\title{
DIELECTRIC VARIABILITY OF A 130 m ANTARCTIC ICE CORE: IMPLICATIONS FOR RADAR SOUNDING
}

\author{
by \\ J.C. Moore \\ (British Antarctic Survey, Natural Environment Research Council, \\ High Cross, Madingley Road, Cambridge CB3 OET, England)
}

\section{ABSTRACT}

A $130 \mathrm{~m}$ long ice core has been dielectrically profiled. From an analysis of the measurements, we obtain a profile of the high-frequency (radio-echo) conductivity. This profile has been represented by a simplified 700 layer model. The model has layers of differing conductivities, permittivities and thicknesses. A reflection-coefficient log can be calculated, assuming that permittivity is a smooth function of depth. Variations in conductivity are shown to be more likely sources of internal reflections from depths greater than a few hundred metres than changes in permittivity caused by density changes. The $\log$ is convoluted with input pulses of various frequencies and pulse lengths in order to produce synthetic radargrams. These show features that correspond to the internal reflections typically seen when radio echo-sounding polar ice sheets.

\section{INTRODUCTION}

When radar sounding polar ice sheets, internal echoes from within the ice are often detected. Some of these echoes have tentatively been correlated with dates of volcanic eruptions (Millar 1982). The current measured in high-field direct-current profiling (ECM) is found to be correlated with acidity (Hammer 1980), with peaks in current found particularly in ice from years following volcanic eruptions. Variations in admittance at radar-sounding frequencies are the cause of internal echoes, and may be due to conductivity changes at d.c. or to changes in dielectric absorptions.

The analysis described here is based on measurements of permittivity and conductivity through the frequency range $20 \mathrm{~Hz}-300 \mathrm{kHz}$. The values of conductivity at the highest frequencies measured are relevant to radio echosounding if there are no dielectric dispersions between the frequency range measured and that employed in radio echosounding.

Previous attempts to model a dielectrically layered ice sheet have been hampered by lack of experimental measurements on ice cores. Instead, calculations have inferred changes in permittivity from spot measurements of density, using empirical relationships (Ackley and Keliher 1979).

Using a new rapid dielectric profiling system (Moore and Paren 1987), the electrical properties of a $130 \mathrm{~m}$ long ice core have been analysed at a resolution of $5 \mathrm{~cm}$. This length is small compared to an annual accumulation of $0.4 \mathrm{~m}$ ice, thus allowing seasonal variability to be investigated.

The core was drilled in 1985-86 near the summit of Dolleman lsland $\left(70^{\circ} 35^{\prime} \mathrm{S}, 60^{\circ} 55^{\prime} \mathrm{W}\right)$, on the east coast of the Antarctic Peninsula. The in-situ temperature of the ice was $-17^{\circ} \mathrm{C}$. The dielectric profiling in the U.K. was carried out in cold-rooms at $-20^{\circ} \mathrm{C}$. All measurements on the ice were made through a polythene sleeve which had protected the core since its retrieval at the time of drilling.

The core is only $130 \mathrm{~m}$ long and therefore is not typical of ice from polar ice sheets, which are usually kilometres thick. The high-frequency conductivity of ice depends on both its density and its chemistry. The conductivity changes found are much greater than those expected from density contrasts and are therefore presumably the effect of chemical changes. Chemical content is unaffected by densification, except for slow diffusion processes. The high-frequency permittivity of ice is mainly a function of density. Therefore in this paper we study the effects of conductivity contrasts rather than permittivity contrasts, because of the relatively greater importance of the former effect when echo-sounding deep ice sheets.

\section{REFLECTION-COEFFICIENT CALCULATION}

The core within its polythene sleeve, sandwiched between the electrodes of the profiling system, is equivalent to an ice sample in series with two blocking electrodes. The dielectric behaviour of an ice-blocking electrode configuration is represented as a small number of overlapping simple Debye dispersions with relaxation times $\tau_{\mathrm{i}}$ and dispersion strengths $\Delta \varepsilon_{\mathrm{i}}$. The complex capacitance $C^{*}$ of the system is then

$$
C^{*}=C_{\mathrm{AIR}}\left(\varepsilon_{\infty}^{\prime}+\sum_{i=1}^{m} \Delta \varepsilon_{\mathrm{i}} /\left(1+j \omega \tau_{\mathrm{i}}\right)\right]
$$

where $\varepsilon_{\infty}^{\prime}$ is the high-frequency permittivity of the system, $\omega$ is the angular frequency, $j=(-1)^{\frac{1}{2}}$, and $C_{\mathrm{AIR}}$ is the measured capacitance between the electrodes, ${ }_{\text {with }}$ ice replaced by air. Notice that there is no d.c.-conductivity term in Equation (1), due to the presence of the blocking layer. In practice the bridge measures the real part of the capacitance and the real part of the conductance. The details of how the dielectric parameters of ice (including its d.c. conductivity) are found from measurements on the blocking-layer capacitor are given in Gross and others (1980). The high-frequency limit of conductivity of the ice, $\sigma_{\infty}$, can be found from the complex capacitance as

$$
\sigma_{\infty}=G_{\infty} \varepsilon_{0} Q / C_{\mathrm{AIR}}(1+Q)
$$

and the high-f requency limit of permittivity of the ice, $\varepsilon_{\infty}$, is given by $\varepsilon_{\infty}=\varepsilon_{\infty}^{\prime} Q /\left((1+Q)-\varepsilon_{\infty}^{\prime} / \varepsilon_{1}\right)$ where $Q$ is the average ratio of the thickness of the ice to the blockinglayer thickness between the ice and electrodes, $G_{\infty}$ is the measured high-frequency limit of the conductance of the system, $\varepsilon_{0}$ is the permittivity of free space, and $\varepsilon_{1}$ is the dielectric constant of the blocking layer.

The highest frequencies used are well above the frequency corresponding to the shortest relaxation time thought to occur in natural ice. Thus good estimates of $\sigma_{\infty}$ and $\tan \delta\left(=\sigma_{\infty} / \omega \varepsilon_{0} \varepsilon_{\infty}\right)$ are obtained for the VHF range, and can therefore be used to determine changes in admittance of ice that are relevant to radio echo-sounding. This is supported by evidence quoted by Glen and Paren (1975), where measurements of conductivity of Greenland ice made by Paren at $100 \mathrm{kHz}$ are very close to measurements made 
by Westphal at $150 \mathrm{MHz}$. Millar (unpublished) used Westphal's conductivity measurements to calculate the radar absorption in natural Antarctic ice. He found excellent agreement between calculated attenuation and known attenuation measurements of radar reflections from a subglacial lake near Vostok Station. These two results are not conclusive evidence that there are no dielectric dispersions above $100 \mathrm{kHz}$, since Westphal measured only a few samples, and Millar's calculation depends greatly on the attenuation-temperature relationship used. However, there is evidence that high-frequency dielectric dispersions occur in laboratory-grown ice (Johari 1976).

The reflection coefficient is calculated, following Ackley and Keliher (1979). The characteristic bulk impedance $Z_{i}$ of layer $i$ is defined by

$$
Z_{i}=\frac{j \mu_{0} \omega}{\gamma_{i}}
$$

where $\mu_{0}$ is the magnetic permeability of free space and $\gamma_{i}$ is the propagation constant

$$
\gamma_{i}^{2}=\varepsilon_{0} \mu_{0} \varepsilon_{i} \omega^{2}+j \mu_{0} \sigma_{i} \omega
$$

where $\varepsilon_{i}$ and $\sigma_{i}$ are the relative dielectric constant and conductivity of the layer.

The reflection amplitude $r_{\mathrm{i}}$ at a boundary within the medium is given by

$$
r_{\mathrm{i}}=\frac{Z_{i-1}-\hat{Z}_{i}}{Z_{i-1}+\hat{Z}_{i}}
$$

where $Z_{i-1}$ is the characteristic bulk impedance of the $i-1$ st layer and $\hat{Z}_{i}$ is the surface impedance of the $i$ th layer.

$$
\hat{z}_{i}=z_{i} \frac{\hat{z}_{i+1}+z_{i} \tanh \gamma_{i} h_{i}}{z_{i}+\hat{z}_{i+1} \tanh \gamma_{i} h_{i}}
$$

where $h_{i}$ is the thickness of the layer $i$. then

If the change in impedance between any layer is small,

$$
r_{\mathrm{i}} \simeq \frac{z_{i-1}-z_{i}}{z_{i-1}+z_{i}}
$$

If the change in impedance is due solely to permittivity changing from $\varepsilon$ to $\varepsilon+\Delta(\varepsilon)$, then Paren and Robin (1975) give the power-reflection coefficient

$$
R=|r|^{2}=\left|\frac{\ddagger \Delta(\varepsilon)}{\varepsilon}\right|^{2}
$$

If the change in impedance is due solely to a change in conductivity, then

$$
r_{\mathrm{i}}=\frac{j \mu_{0} \omega\left[\sigma_{i}-\sigma_{i-1}\right]}{\gamma_{i}^{2}+2 \gamma_{i} \gamma_{i-1}+\gamma_{i-1}^{2}}
$$

let $A=\varepsilon_{0} \mu_{0} \varepsilon \omega^{2}$

$$
r_{\mathrm{i}}=\frac{j \mu_{0} \omega\left(\sigma_{i}-\sigma_{i-1}\right)}{2 A+j \mu_{0} \omega\left(\sigma_{i}-\sigma_{i-1}\right)+2\left[A^{2}+j A \mu_{0} \omega\left(\sigma_{i}+\sigma_{i-1}\right)-\mu_{0}^{2} \sigma_{i} \sigma_{i-1} \omega^{2}\right]^{\frac{1}{2}}}
$$

If $\varepsilon_{0} \varepsilon \omega \gg \sigma$, then

$$
r_{\mathrm{i}} \simeq \frac{j\left[\sigma_{i}-\sigma_{i-1}\right]}{4 \varepsilon_{0} \varepsilon \omega}
$$

The same result can be derived from equation (3) in Paren (1981).
The condition $\varepsilon_{0} \varepsilon \omega \gg \sigma$ is equivalent to $\varepsilon \gg \tan \delta$ and is generally true when radar sounding ice sheets, for instance at $60 \mathrm{MHz}, \varepsilon \sim 3$ and $\tan \delta \sim 0.003$.

The factor $j$ merely causes a constant phase delay in all reflections from boundaries of $\pi / 2$, or equivalently a change in receiving-antenna height above the surface. Therefore the amplitude-reflection coefficient due solely to loss tangent varying from $\tan \delta$ to $\tan \delta+\Delta(\tan \delta)$ across a boundary is

$$
r=\frac{1}{4} \Delta(\tan \delta)
$$

Equations (3) -(5) show that small percentage changes in permittivity will produce larger reflection coefficients than a similar percentage change in $\tan \delta$. However, the estimate of $\varepsilon_{\infty}^{\prime}$ in Equation (1), from data in the frequency range studied, is not sufficiently precise to allow it to be determined to better than a few per cent. At the same time there may be additional errors in the estimate of $\varepsilon_{\infty}$ if there are relaxation processes at $\mathrm{MHz}$ frequencies like those observed by Johari (1976) and von Hippel and others (1972) in the dielectric spectrum of some laboratory-grown ice samples. They found dispersions with $\varepsilon \sim 0.1$. Large changes in conductivity are easy to observe and are obtained straightforwardly from measurements of $G_{\infty}$, using Equation (2); conductivity is relatively insensitive to $Q$ and to the permittivity of the blocking layer. Peaks in conductivity of up to seven times the normal value are found in small sections of the core. Conductivity contrasts shown in Figure 1 can produce power-reflection coefficients of about $-57 \mathrm{~dB}$. Paren and Robin (1975) calculate reflection coefficients for a discontinuous density change which causes a change in high-frequency permittivity across a boundary The density change corresponds to a difference in density between bubble-free ice of density $0.92 \mathrm{Mg} \mathrm{m}^{-3}$ and bubbly ice at a density dependent on overburden pressure. The overburden pressure required to produce a density contrast which gives rise to a reflection coefficient of $-57 \mathrm{~dB}$ is approximately 18 bar. This corresponds to a depth of about $250 \mathrm{~m}$ in the ice sheet. Below this depth the density contrast decreases, giving smaller reflection coefficients. Since ice sheets are typically several kilometres in thickness, conductivity contrasts are probably the dominant effect for both the polar ice sheets. The conductivity log (that is, the profile of high-frequency conductivity) was therefore used to compute reflection coefficients. No reflection coefficients due to changes in permittivity are used; permittivity is assumed to vary with core density according to Looyenga's model (see equation (9) in Glen and Paren (1975)):

$$
\varepsilon_{\infty}=(1+0.469 v)^{3}
$$

where $U$ is the density of firn/density of ice. This relationship gives a permittivity of 3.17 for ice, equivalent to a velocity for radar waves of $168.5 \mathrm{~m} \mathrm{ss}^{-1}$.

A 695 layer model was constructed from the 2500 dielectric spectra from which the conductivity log was derived. The thickness of each layer varied from $5 \mathrm{~cm}$ to over $1 \mathrm{~m}$, as shown in Figure 1. Each layer consists of ice for which the variations between adjacent measurements do not exceed $0.4 \times 10^{-5} \mathrm{~S} \mathrm{~m}^{-1}$, equivalent to $20 \%$ of the average value of the conductivity. A change in conductivity from $2 \times 10^{-5} \mathrm{~S} \mathrm{~m}^{-1}$ to $2.4 \times 10^{-5} \mathrm{~S} \mathrm{~m}^{-1}$ would produce a power-reflection coefficient, $R,\left(R=|r|^{2}\right)$ at 60 $\mathrm{MHz}$ of $8.94 \times 10^{-9}$, or about $-80 \mathrm{~dB}$. by

The travel time of radio waves in each layer is given

$$
t t=h\left[\varepsilon_{\infty}\right]^{\frac{1}{2}} / c
$$

where $h$ is the thickness of the layer, and $c$ is the velocity of light in free space.

The amplitude-reflection coefficient and two-way travel times for the primary reflection from each of the boundaries were calculated, using Equations (3), (5) and (6).

The next step is to calculate the response of any pulse to this reflection-coefficient/travel-time log through the coherent superpositions of reflections. We call the response a synthetic radargram. To create the radargram an input pulse must be convoluted with the reflection-coefficient log. 


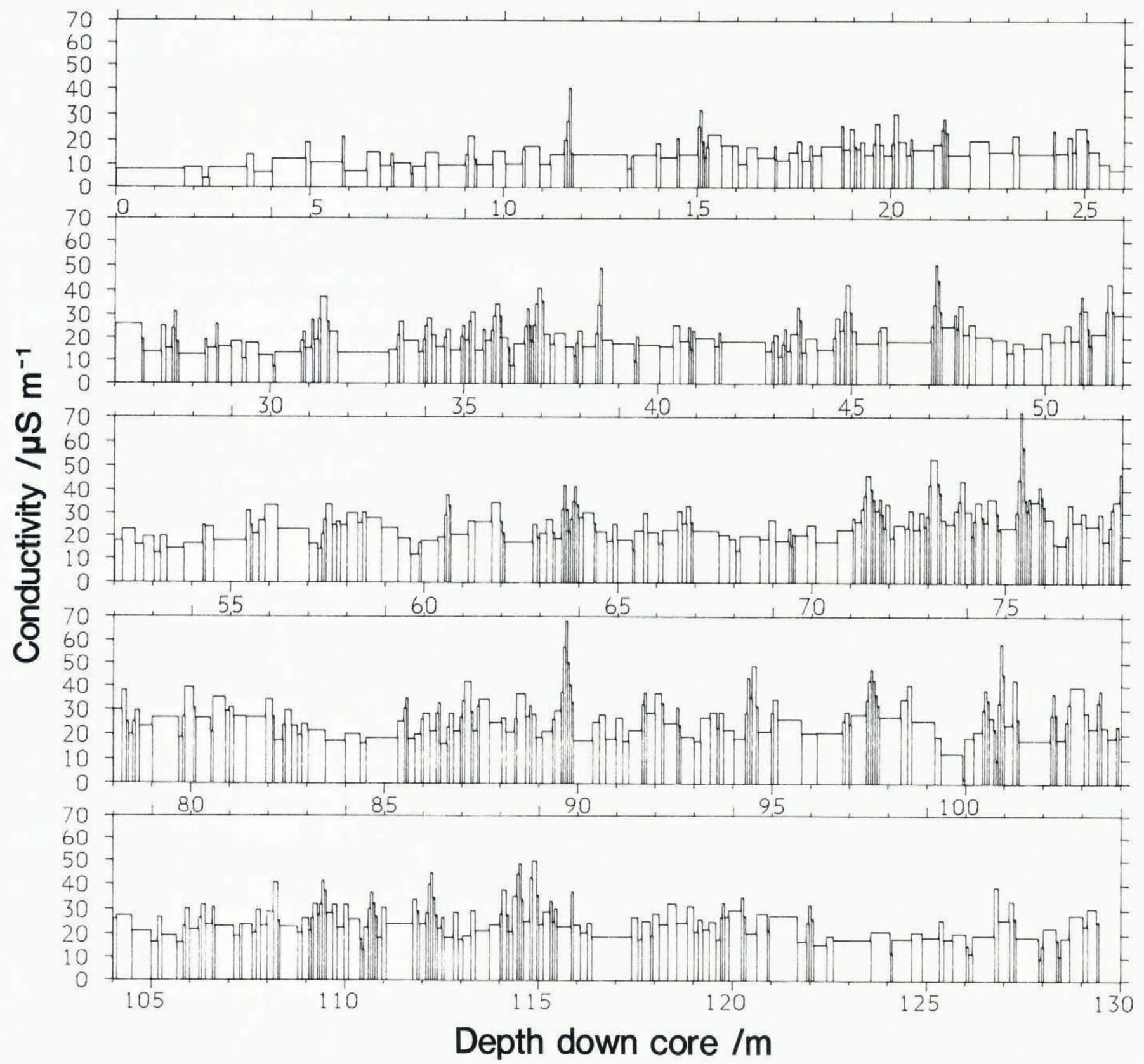

Fig. 1. The 695 layered high-frequency conductivity log.

The input pulses chosen all have the general form

$$
\psi(t)=\frac{2 \sin (\omega t+\beta)}{\exp \left(-4 t^{\prime} / T\right)+\exp \left(4 t^{\prime} / T\right)}
$$

where $T$ is the pulse length, $\omega$ is angular frequency, $\beta$ is phase angle, $t$ is time, and $t^{\prime}=t-T / 2$.

The exponential terms provide a symmetrical envelope to the pulse.

To simulate an "envelope-detected" output, as used in real radar sounding, two input pulses are chosen: $\psi_{1}$, with $\beta=0$, and $\psi_{2}$ with $\beta=\pi / 2$. The two pulses are convoluted with the amplitude-reflection/travel-time log and synthetic radargrams found. The two radargrams produced by the convolutions are squared and added to give the pseudo"envelope-detected" radargram (Nye and Berry 1974).

The results for various input pulses of different frequencies and pulse lengths are shown in Figure 2. Losses due to geometric spreading and electromagnetic absorption are not included. Geometric spreading losses, for example, depend greatly on the height above the surface of the radio-echo antenna. The attenuation due to absorption in ice is $18 \mathrm{~dB} \mathrm{~km}^{-1}$ for an average ice-core conductivity of
$2 \times 10^{-5} \mathrm{~S} \mathrm{~m}^{-1}$. For the deepest reflection, at a depth of $130 \mathrm{~m}$, this additional loss is $4.8 \mathrm{~dB}$. Multiple internal reflections produce reflection coefficients with magnitudes about one-millionth of primary reflections, and are therefore not considered in the analysis.

\section{DISCUSSION}

Despite the large number of layers in the model, there are relatively few large reflections in the synthetic radargram. As may be seen in Figure 2, both the magnitudes and shapes of the peaks in the synthetic radargrams change with frequency and pulse length. As expected, a shorter pulse length gives a greater resolution but a lower magnitude of response; this can be seen in the response for an input frequency of $35 \mathrm{MHz}$ with pulse lengths of 60 and $150 \mathrm{~ns}$. The peaks in the response to the $150 \mathrm{~ns}$ pulse are offset slightly because of the longer time required for the maximum input-pulse amplitude to reach any depth. Increasing the input frequency reduces the response intensity, since changes in $\tan \delta$ govern the reflection coefficients, and $\tan \delta$ is inversely proportional to frequency. The thicknesses of the layers used in the model 

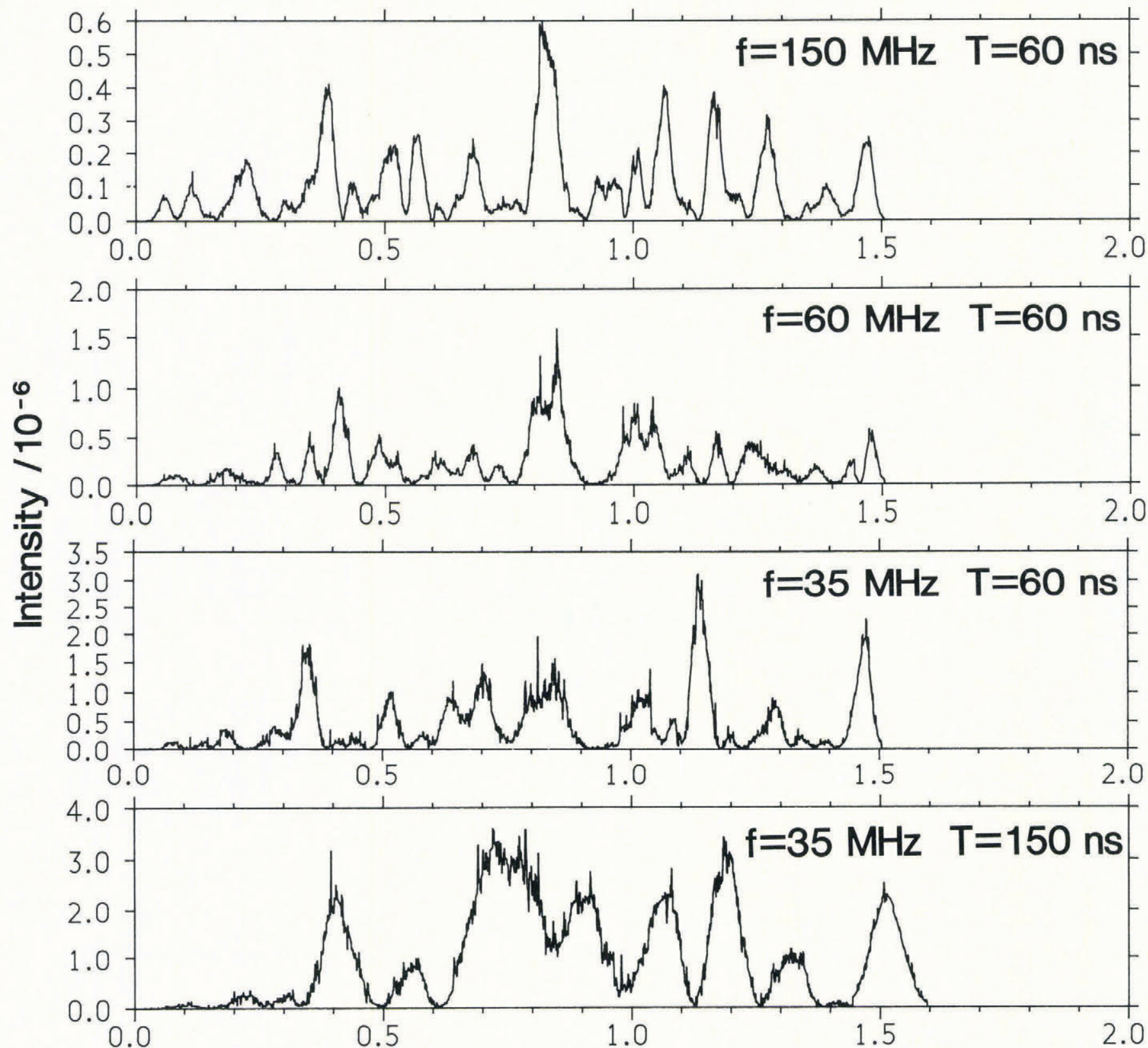

\section{Time / $\mu \mathrm{s}$}

Fig. 2. Synthetic radargrams for input pulses of various frequencies, $f$, and pulse lengths, $T$. The response is normalized for an input pulse of unit intensity.

are an appreciable fraction of the radar wavelength in ice. The magnitude of the reflections from the layer depends on the interference of the waves reflected from both boundaries of the layer. If the thickness of a layer depends on local accumulation rate, then the response of the ice to input pulses of one frequency may vary greatly with small changes in antenna position. This may explain why real reflecting horizons are neither continuous nor perfectly flat when studied in detail.

The largest event, at about $0.8 \mu \mathrm{s}$ two-way travel time, corresponds to the largest peaks in conductivity, around $75 \mathrm{~m}$ depth down the core. The reflection coefficient of the $0.8 \mu \mathrm{s}$ peak is about $-60 \mathrm{~dB}$, a typical value for internalreflection horizons. For example, Paren and Robin (1975) quoted values for many horizons in Greenland and Antarctica, observed at 35 and $60 \mathrm{MHz}$, that are between -60 and $-83 \mathrm{~dB}$, after correction for the effects of geometric spreading and absorption.

The reflection coefficient given by Equations (3) and (5) is only true if the boundary between the two layers is sharp. This is so. The conductivity log displayed in Figure 1 often shows a very sudden change in conductivity, typically a factor of 4 , in only $5-10 \mathrm{~cm}$ at the peaks. The down-core side of the peak is usually even more abrupt. This is narrow compared with typical radio-echo wavelengths in ice $(\sim 2.7 \mathrm{~m}$ at $60 \mathrm{MHz})$, so a sharp boundary is probably an appropriate model. The chemical stratigraphy of the core also exhibits quite sudden changes between adjacent samples (personal communication from R. Mulvaney). Equation (5) is valid for small relative changes in admittance; a change of $6 \times 10^{-5} \mathrm{~S} \mathrm{~m}^{-1}$ produces a change in admittance of $1 \%$ at $35 \mathrm{MHz}$, so Equation (5) is appropriate.

Unless conductivity variations are invoked it is difficult to explain the existence of internal layers in thick ice sheets (Paren and Robin 1975). Near the surface, ice lenses or more extensive features of melt or depth hoar may cause local changes in permittivity that are great enough to give large reflection coefficients. Density contrasts are smoothed out rapidly with depth, and a number of theories for admittance changes in ice have been suggested (Ackley and Keliher 1979). Conductivity variations like those measured in the Dolleman Island core are of the correct character to explain the results of radio echo-sounding. 
The inverse problem that has long been of interest is the calculation of the permittivity and conductivity profiles from the radargram of an ice sheet. To do this by a deconvolution of the radargram would require detailed information on the input wave in the ice. This has so far not been available. A unique solution in terms of conductivity and permittivity variations is probably not possible. Walford (1986) discussed the equipment required for a deconvolution. A record of both phase and amplitude of the echo would be needed rather than the photographically recorded intensity traces in current use. A radar sounder which gives a very high signal-to-noise ratio over as wide a bandwidth as possible is essential. The best that may be achieved with present echo sounders is the detection of large peaks in conductivity or permittivity such as those caused by some volcanic events (Millar 1982). The smaller events and any annual variation in properties are likely to be undetectable.

\section{ACKNOWLEDGEMENTS}

I thank M.E.R. Walford for providing the incentive to write this paper, and K.W. Nicholls, J.G. Paren, H.F.J. Corr, D.G. Vaughan and G.W. Gross for much helpful discussion and encouragement during its preparation. I also thank the drilling team on Dolleman Island (D.A. Peel, R. Mulvaney, S. Cooper and B.M. Davison) who retrieved the core.

\section{REFERENCES}

Ackley, S.F., and T.E. Keliher. 1979. Ice sheet internal radio-echo reflections and associated physical property changes with depth. J. Geophys. Res., 84(B10), 56755680 .

Glen, J.W., and J.G. Paren. 1975. The electrical properties of snow and ice. J. Glaciol., 15(73), 15-38.

Gross, G.W., I. Cox Hayslip, and R.N. Hoy. 1980. Dielectric relaxation spectrum of ice measured with linear blocking electrodes. Geophysics, 45(5), 914-927.

Hammer, C.U. 1980. Acidity of polar ice cores in relation to absolute dating, past volcanism, and radio-echoes. $J$. Glaciol., 25(93), 359-372.

Hippel, A. von, R. Mykolajewycz, A.H. Runck, and W.B. Westphal. 1972. Dielectric and mechanical response of ice $\mathrm{I}_{\mathrm{h}}$ single crystals and its interpretation. J. Chem. Phys., 57(6), 2560-2571.

Johari, G.P. 1976. The dielectric properties of $\mathrm{H}_{2} \mathrm{O}$ and $\mathrm{D}_{2} \mathrm{O}$ ice $\mathrm{I}_{h}$ at $\mathrm{MHz}$ frequencies. J. Chem. Phys., 64(10), 3998-4005.

Millar, D.H.M. 1982. Acidity levels in ice sheets from radio echo-sounding. Ann. Glaciol., 3, 199-203.

Millar, D.H.M. Unpublished. Radio echo layering in polar ice sheets. (Ph.D. thesis, University of Cambridge, 1981.)

Moore, J.C., and J.G. Paren. 1987. A new technique for dielectric logging of Antarctic ice cores. J. Phys. (Paris), 48, Colloq. C1, 155-160. (Supplément au 3.)

Nye, J.F., and M.V. Berry. 1974. Dislocations in wave trains. Proc. R. Soc. London, Ser. A, 336, 165-190.

Paren, J.G. 1981. Correspondence. Reflection coefficient at a dielectric interface. J. Glaciol., 27(95), 203-204.

Paren, J.G., and G. de Q. Robin. 1975. Internal reflections in polar ice sheets. J. Glaciol., 14(71), 251-259.

Walford, M.E.R. 1986. Scattering of radio waves by polar ice sheets. In Uscinski, B.J., ed. Wave propagation and scattering. Oxford, Clarendon Press, 261-279. (The Institute of Mathematics and Its Applications Conference Series. New Series 5.) 